\title{
Health-related quality of life using QOLIE-31: Before and after epilepsy surgery a prospective study at a tertiary care center
}

\author{
Faiz Uddin Ahmad, Manjari Tripathi*, M. V. Padma*, Shailesh Gaikwad**, Aditya Gupta, C. S. Bal ${ }^{* * *}$, \\ Chitra Sarkar", Surya Gupta^, Ashima N. Wadhawan^, Bhavani S. Sharma, Poodipedi Sarat Chandra
}

Departments of Neurosurgery, *Neurology, ${ }^{* *}$ Neuroradiology and ${ }^{* *}$ Nuclear Medicine, ${ }^{*}$ Neuropathology and ^Clinical Psychology, Neurosciences Center, All India Institute of Medical Sciences, New Delhi - 110 029, India

\begin{abstract}
Background: Outcome following epilepsy surgery has traditionally been measured in terms of relief of seizures. However, changes in health-related quality of life (HRQOL) after surgery for intractable epilepsy are also important to document. There are no studies on the Indian population which assess the outcome of epilepsy surgery in terms of HRQOL. Materials and Methods: We conducted a prospective study on the patients undergoing epilepsy surgery for intractable seizures, between February 2004 and May 2006 at our center. All patients cleared for epilepsy surgery by
\end{abstract} the epilepsy surgery team were taken up for study. All patients $<15$ years age and mentally retarded or with progressive neurological diseases were excluded. Demographic profile, seizure characteristics and seizure outcome using Engel grading was assessed. Health-related quality of life was assessed using QOLIE-31 questionnaire before surgery and six months after surgery. Results: Thirty-six patients satisfying the inclusion/exclusion criteria were included in the analysis. Twenty-nine of these (Group 1) had good seizure outcome (Engel 1 and 2), while seven patients (Group 2) had poor seizure outcome (Engel 3 and 4 ) at six months. Overall, $77 \%$ of all study patients were completely seizure-free at follow-up. There was no baseline difference in the seven domains of QOLIE-31 between the two groups. There was very significant improvement ( $P$ value $>0.005$ using paired sample $\mathrm{T}$ test) in all the domains of QOLIE-31 in the good outcome group after surgery. Health-related quality of life improvement was seen in all the domains in the poor outcome group also, however, it was statistically significant only for the following parameters: seizure worry, overall QOL, emotional wellbeing, energy fatigue and social functioning domains. Improvement in seizure worry, overall QOL, emotional wellbeing and social functioning was significantly more in Group 1 as compared to Group 2. Conclusion: Complete seizure-free state after surgery is associated with very significant improvement in HRQOL parameters. Several, but not all parameters of HRQOL as assessed by QOLIE-31, improved after surgery even in the poor seizure outcome group. The improvement in domains of seizure worry, overall QOL, emotional wellbeing and social functioning is significantly more in those patients in whom complete seizure-free state is achieved.

Key words: Epilepsies, partial/surgery or epilepsy, psychiatric status rating scales or quality of life or severity of illness index or sickness impact profile, temporal lobe/surgery

Epilepsy is both a medical diagnosis and a social stigma, especially in developing countries like India. The possibility of recurrent seizures remains a silent, chronic daily hazard for most epilepsy patients. The stigma and underlying worry about epilepsy are not usually considered in the follow-up of patients with few or no recent seizures. Low self-esteem, lack of independence, need for antiepileptic drugs (AEDs), restrictions on driving etc are chronic problems frequently faced by patients with epilepsy. ${ }^{[1,2]}$

Epilepsy surgery offers an important treatment option for a subset of carefully selected patients with intractable seizures. Outcome following epilepsy surgery has traditionally been measured in terms of relief of seizures. This has been determined by focusing on four broad areas postoperatively:

1 Changes in seizure-related variables like type, frequency etc.

2. The amount and number of AEDs needed.

3. Assessment of morbidity and mortality.

4. Changes in neuropsychological evaluation.

It is certainly very important to assess the above outcome parameters to establish the effectiveness of epilepsy surgery, however, these may not provide the 
total picture as to how patient perceives the change in his/ her social functioning after surgery. It is thus equally important to evaluate the efficiency of epilepsy surgery in terms of health-related quality of life (HRQOL) assessment. Health-related quality of life is a function of the conceptual distance between 'how I am now' and 'how I would like to be', the gap between actual and expectation. This is known as 'Calman's gap', since Calman suggested that the key aim of medical care (which includes medical/surgical treatment of epilepsy) should be to narrow the gap between the patients' hopes/ expectations and the patients' current state. ${ }^{[3]}$

There are no studies on the Indian population which assess the outcome of epilepsy surgery in terms of HRQOL. It cannot be overemphasized that epilepsy surgery is a costly proposition. It also places the patients and the relatives under a lot of psychological and mental stress. The presurgical investigation is exhaustive and the patient has to be carefully monitored for the antiepileptic medication after surgery for a prolonged period. It is thus important to assess objectively whether the good seizure outcome does translate into improved health-related quality of life. It was with this aim that this prospective study was undertaken.

\section{Materials and Methods}

We conducted a prospective study on the patients undergoing epilepsy surgery for intractable seizures, between February 2004 and May 2006, in the Department of Neurosurgery, Neurosciences Centre, All India Institute of Medical Sciences, New Delhi.

\section{Inclusion criteria}

All patients cleared for epilepsy surgery by the epilepsy surgery team of the Neurosciences Center, All India Institute of Medical Sciences were taken up for study.

\section{Exclusion criteria}

All patients 15 years of age or younger, mentally retarded or with progressive neurological diseases were excluded from the study.

\section{Presurgical evaluation}

The presurgical evaluation of potential candidates included a detailed history and clinical examination, EEG, long-term video EEG and high-quality MRI scan (including T1 weighted inversion recovery sequences and FLAIR sequences in oblique coronal planes 3 mm thick, perpendicular to the principal axis of the hippocampal formation and sensitive enough to detect subtle alterations in hippocampal size, morphology and signal intensity). Gadolinium enhancement was used if structural pathology was suspected. An interictal SPECT study was done using HMPAO. In doubtful cases, an ictal SPECT was done using Tc-ECD.

\section{Surgical procedures}

For mesial temporal sclerosis (MTS), a standard anteromedial temporal lobectomy $(3.5 \mathrm{~cm}$ on the dominant and $5.5 \mathrm{~cm}$ on the non-dominant side) with a subpial amygdalohippocampectomy was performed. For lesional temporal lobe and extratemporal epilepsy, lesionectomy under electrocorticographic guidance was performed.

\section{Seizure outcome assessment}

After discharge, follow-up visits were scheduled at two weeks, one month, three months, six months and every six months thereafter. Antiepileptic drugs were continued for one year and then tapered thereafter. Seizure outcome was assessed using Engel's grading ${ }^{[4]}$ at six month follow-up.

\section{HRQOL assessment}

A quality of life questionnaire, QOLIE- $31,{ }^{[5,6]}$ containing seven multi-item scales, including seizure worry, emotional wellbeing, energy/ fatigue, cognition, medication effects, social effects, health status and overall quality of life, was administered to all the patients in the study preoperatively and six months after surgery.

The Quality of life in epilepsy inventory (QOLIE-31) (copyright 1993, RAND) was developed in cooperation with professional postgraduate services, a division of Physicians World Communications Group and the QOLIE Development Group. Written permission to use QOLIE-31 in our study was obtained from RAND, 1700 Main Street, PO Box 2138, Santa Monica, California 90407-2138.

The 31-item QOLIE-31 (Version 1.0) has been derived from the longer QOLIE-89. ${ }^{[2,6]}$ It has been translated into several European languages and has been tested for reliability and validity as a measure of HRQOL. ${ }^{[7]}$

Precoded numeric values to responses to some QOLIE31 items are in a direction such that a higher number reflects a more favorable health state. However, precoded numeric values to some other items on QOLIE-31 are such that a lower score reflects a more favorable state. To account for these differences, the scoring procedure for QOLIE-31 first converts the raw precoded numeric values of items to $0-100$ point score, with higher reflected scores always reflecting better quality of life. $\mathrm{T}$ scores can be determined for each of theses $0-100$ point scale scores using the keys in QOLIE-31. These $\mathrm{T}$ scores represent linear transformations of the scores that produce a mean of 50 and standard deviation of 10 for a cohort of 304 adults with epilepsy (thus a person with a $\mathrm{T}$ score of 50 has a score equal to the mean of 
the epilepsy cohort). ${ }^{[5]}$

\section{Statistical analysis}

The data was analyzed using commercially available SPSS 11.5 software. T-scores of each QOLIE-31 domain (seizure worry, emotional wellbeing, energy/ fatigue, cognition, medication effects, social effects, health status and overall quality of life) were used for final analysis. Paired-samples T test was used to look for significant improvement in patients after epilepsy surgery. Independent-samples $\mathrm{T}$ test was applied to look for significant changes in QOL parameters between good and poor seizure outcome groups. Binary logistic regression analysis was applied using Engel good and bad outcomes as dependent variables, to check for its dependence on different seizure parameters and clinical profile. To find the difference in numbers of patients reporting worse 'medication effects' after surgery in the two groups, the chi square test was applied.

\section{Results}

Thirty-six patients satisfying the inclusion/exclusion criteria were included in the analysis. Demographic data and seizure variables are shown in Table 1. Twenty-nine of these (Group 1) had good seizure outcome (Engel 1 and 2), while seven patients (Group 2) had poor seizure outcome (Engel 3 and 4) at six months. Among the patients in the good seizure outcome group, all except one had Engel 1a outcome (completely seizure-free since surgery with no auras). One patient had one seizure in the postoperative period. One patient in Group 2 had Engel Grade 4 outcome. Sixteen patients in Group 1 had MTS, eight had temporal lesions (three- cortical dysplasia, three- cavernoma, one- DNET, one- gliosis) and five had extratemporal lesions (one- parietal DNET, one- frontal gliosis, one- bilateral occipital gliosis, one- frontal DNET, one- parieto-occipital cortical dysplasia). In Group 2, two patients had MTS, three had temporal lesions (two- DNET, onw- cortical dysplasia) and two had extratemporal lesions (one- left frontal gliosis, oneright parietal cortical dysplasia).

All operated tissue was subjected to histopathological examination. Apart from one case each of hemiplegia and transient facial paresis, the operative procedures were free of complications. There was no mortality in our series. Overall, $77 \%$ of all study patients were completely seizure-free at follow-up. Complete seizure freedom was achieved in $88.9 \%$ of patients with MTS, $63.6 \%$ of patients with temporal lesions and $71 \%$ of those with extratemporal lesions.

In the overall study group, the average duration of seizures was 12.6 years, with a mean frequency of 34 seizures per month. Seizure profile of patients in both the groups is shown in Table 1 . The average seizure duration in Group 1 and 2 was 12 years and 15.7 years respectively, which showed no statistical difference. There was, however, a significant difference between the frequency of seizures in both groups $(21 / \mathrm{month}$ and $85 /$ month respectively, $\mathrm{P}$ value $=0.01$ ). The same significant difference persisted when the frequency was categorized into four groups i.e., < five/month, 6-29/ month, one to five/day and > five/day. In Group 1, VEEG, MRI and SPECT were concordant with histopathology in 23 cases $(79 \%)$, whereas the same was true in five cases in Group 2 (74\%). This inter-group difference was not significant.

The HRQOL measures are shown in Table 2. There was no baseline difference in the seven domains of QOLIE-31 between the two groups, implying that the preoperative QOL was same in both the groups. There was very significant improvement $(P$ value $>0.005$ using paired sample T test) in all the domains of QOLIE-31 in the good outcome group after surgery. Health-related

\begin{tabular}{|c|c|c|}
\hline \multicolumn{3}{|c|}{ Table 1: Clinical profile and seizure variables } \\
\hline & Group 1 (Good seizure outcome) n=29 & Group 2 (Poor seizure outcome) $n=7$ \\
\hline Age (Mean \pm SD) & $25.59 \pm 6.3$ & $25.0 \pm 4.3$ \\
\hline Gender (Male/female) & $23 / 6$ & $4 / 3$ \\
\hline Duration of seizures in years (mean $\pm S D)$ & $11.97 \pm 5.0$ & $15.71 \pm 3.63$ \\
\hline \multicolumn{3}{|l|}{ Duration of seizures: } \\
\hline$<10$ years & 14 & 0 \\
\hline$\geq 10$ years & 15 & 7 \\
\hline Frequency of seizure per month (mean $\pm \mathrm{SD}$ ) & $21.36 \pm 66.96$ & $85.29 \pm 161.91$ \\
\hline \multicolumn{3}{|l|}{ Frequency } \\
\hline$<5 /$ month & 20 & 1 \\
\hline 6-29/month & 5 & 2 \\
\hline $1-5 /$ day & 3 & 3 \\
\hline$>5 /$ day & 1 & 1 \\
\hline Side of surgery (right/left) & $20 / 9$ & $6 / 1$ \\
\hline Concordance of pre-op investigations & $23(79 \%)$ & $5(71 \%)$ \\
\hline \multicolumn{3}{|l|}{ (VEEG, MRI, SPECT) with HPE } \\
\hline \multicolumn{3}{|l|}{ Diagnosis } \\
\hline MTS: & 16 & 2 \\
\hline Temporal lesion: & 8 & 3 \\
\hline Extra-temporal lesion: & 5 & 2 \\
\hline
\end{tabular}


Table 2: Health-related quality of life measures

\begin{tabular}{|c|c|c|c|c|c|c|}
\hline QOLIE-31 domains & $\begin{array}{l}\text { Seizure } \\
\text { outcome }\end{array}$ & $\begin{array}{l}\text { Pre-op mean } \\
\text { score }\end{array}$ & $\begin{array}{l}\text { Post-op mean } \\
\text { score }\end{array}$ & $\begin{array}{l}P \text { value (comparing } \\
\text { pre and post } \\
\text { op scores } \\
\text { in each group }\end{array}$ & $\begin{array}{l}\text { Change in mean } \\
\text { score after } \\
\text { surgery }\end{array}$ & $\begin{array}{l}P \text { value (comparing } \\
\text { change in mean } \\
\text { scores between } \\
\text { Group } 1 \text { and } 2 \text { ) }\end{array}$ \\
\hline Seizure & Group 1 & 41.3 & 58.9 & 0.000 & 17.2 & 0.005 \\
\hline Worry & Group 2 & 41.1 & 49.9 & 0.015 & 8.6 & \\
\hline Overall & Group 1 & 37.5 & 58.50 & 0.000 & 19.3 & 0.003 \\
\hline QOL & Group 2 & 38.4 & 48.00 & 0.021 & 9.6 & \\
\hline Emotional & Group 1 & 41.5 & 56.10 & 0.000 & 15.6 & 0.040 \\
\hline Wellbeing & Group 2 & 41.3 & 49.29 & 0.020 & 8.0 & \\
\hline Energy & Group 1 & 47.2 & 53.66 & 0.000 & 6.4 & 0.352 \\
\hline Fatigue & Group 2 & 46.6 & 50.86 & 0.024 & 4.3 & \\
\hline Cognitive & Group 1 & 45.8 & 49.69 & 0.000 & 4.1 & 0.386 \\
\hline Functioning & Group 2 & 44.3 & 46.43 & 0.260 & 2.1 & \\
\hline Medication & Group 1 & 46.6 & 49.93 & 0.007 & 3.3 & 0.906 \\
\hline Effects & Group 2 & 45.4 & 48.43 & 0.253 & 3.0 & \\
\hline Social & Group 1 & 41.1 & 54.80 & 0.000 & 13.6 & 0.033 \\
\hline Functioning & Group 2 & 40.1 & 46.86 & 0.009 & 6.7 & \\
\hline
\end{tabular}

quality of life improvement was seen in all the domains in the poor outcome group also, however, it was statistically significant only in seizure worry, overall QOL, emotional wellbeing, energy fatigue and social functioning domains.

Improvement in several domains of QOLIE-31 was significantly more in Group 1 as compared to Group 2 [i.e., good seizure outcome vs. poor seizure outcome]. In particular, improvement in seizure worry, overall QOL, emotional wellbeing and social functioning was significantly more in Group 1 as compared to Group 2, with maximum difference seen in the 'seizure worry' domain between the two groups ( $P$ value 0.005 ). Improvement in other domains was also more in Group 1 as compared to Group 2, but it did not reach statistical significance. An interesting finding was worsening in 'medication effects' in a higher percentage of Group 1 patients (31\%) as compared to Group 2 patients (14\%), but the difference was not statistically significant.

\section{Discussion}

Surgery for epilepsy can and does have far-reaching impacts which go beyond the relief of seizures. Health-related quality of life refers to the way in which individuals function and their perceived wellbeing in the physical, mental and social domains of life. ${ }^{[6]}$ Because of the potential underutilization of epilepsy surgery, its invasive nature and relatively high costs of presurgical evaluation and subsequent surgery, it is important that the outcome after epilepsy surgery must also be evaluated by HRQOL assessment and its effectiveness be established, especially so in developing countries like India.

The purpose of addressing QOL includes improving the quality of patient care, differentiating among treatment options and evaluating the allocation of healthcare resources. The major domains of QOL are physical, psychological and social, which go beyond the traditional assessment of seizure frequency and severity and the adverse effects of medications.

Health-related quality of life can be assessed by generic or disease-specific measures or a combination of both. Generic instruments assess a broad range of functioning and allow comparisons across patients with different diseases. Disease-targeted instruments concentrate on issues of particular relevance to a specific disease. Several instruments have been devised to assess the quality of life. QOLIE-89, QOLIE-31 and ESI-55 are examples of epilepsy-specific instruments. ${ }^{[6-8]}$

We have used the 31-item QOLIE-31 (Version 1.0) in our study, which has been derived from the longer QOLIE-89. It covers both general and epilepsy-specific domains, with an emphasis on the latter. It consists of seven subscales, grouped under two factors: emotional / psychological effects (seizure worry, emotional wellbeing, overall QOL, energy/fatigue subscales) and medical/social effects (medication effects, work-drivingsocial limitations, cognitive function subscales). It has been translated into several European languages and has been tested for reliability and validity as a measure of HRQOL. ${ }^{[6,7]}$ Though QOLIE-89 is the most comprehensive instrument available, QOLIE-31 is as responsive to issues relevant to epilepsy. ${ }^{[9]}$ It is already more widely used internationally than QOLIE-89, largely because of its brevity, simplicity of scoring and small patient burden in responding to questions. An even shorter version of QOLIE-31, QOLIE-10, has been found useful, both for screening and research. ${ }^{[10]}$ However, QOLIE-31 is preferred where time and resources are available, ${ }^{[10]}$ thus was found to be most suitable for this study. There is no agreed follow-up period for assessment of QOL post surgery. Researchers have chosen periods as diverse as three months, ${ }^{[11]}$ six to eight months, ${ }^{[12]}$ and one year. ${ }^{[13]}$ Longer periods of follow-up are obviously more beneficial but keeping in mind the limitations of 
follow-up in our country, we used six months follow-up for assessing postoperative HRQOL in our study.

There is no simple relationship between seizure duration, seizure frequency and consequences of epilepsy and there is a debate whether a simple reduction (but not complete elimination) of seizures does lead to an improvement in HRQOL. ${ }^{[14-17]}$

Some studies report that good QOL outcome is dependent on being completely seizure-free postoperatively, ${ }^{[12,15,18,19]}$ suggesting that patients with postoperative seizures, even at a reduced frequency, may have worse outcome compared with their preoperative baseline status. ${ }^{[20]}$ This may be because some patients about to go in for epilepsy surgery have high and sometimes even unrealistic, expectations of significant positive changes after surgery. ${ }^{[21]}$ This also affects their perception of QOL after surgery. However, some of these studies used generic measures, which may not be directly applicable to epilepsy surgery patients. To complicate issues further, some old studies reported improved psychosocial functioning after surgery regardless of seizure control. ${ }^{[21,22]}$ More recent studies using epilepsy-specific measures have found that QOL improves in seizure-free patients, but also to a lesser degree in patients having auras only, compared with those with ongoing seizures. ${ }^{[8,23,24]}$ While there is no agreement on the degree of seizure reduction as an outcome measure that affects HRQOL after epilepsy surgery, we observed a significant improvement in QOL using Engel good outcome (Grade 1 and 2) and Engel poor outcome (Grade 3 and 4 ) as parameters for analyzing HRQOL. This suggests that even a few seizures in the postoperative period can significantly impair the quality of life.

The few studies that are available from developing countries on QOL in epileptic patients report poor quality in them. ${ }^{[25,26]}$ Mikati et al. ${ }^{[27]}$ from Lebanon used ESI-55 questionnaire and compared QOL of 20 patients of temporal lobe epilepsy (TLE) who underwent surgery with 17 TLE patients treated medically and 20 healthy volunteers and reported normalization of QOL three years after temporal lobectomy in the surgical group.

We found significant improvement in all parameters of QOLIE-31 after surgery in patients in the good outcome group. It is to be noted that all except one patient in the "good" outcome group were seizure-free and the results for Group 1 might not generalize to patients with Engel $1 \mathrm{~b}$ or Engle 2 outcomes. Several domains of HRQOL improved in patients in the poor outcome group too (see 'results' above). It is noteworthy that the improvement in the domains of seizure worry, overall QOL, emotional wellbeing and social functioning was significantly more in Group 1 as compared to Group 2, with maximum difference seen in the 'seizure worry' domain between the two groups. This implies that the maximum benefit in these parameters is seen in those patients who are seizure-free after surgery. Six out of seven patients in our study in Group 2 had Engel Grade 3 outcome and only one had Grade 4 outcome. This means that even in Group 2, the great majority (87\%) of patients had $>75 \%$ reduction in seizures. This probably explains the improvement in several domains of HRQOL seen after surgery in this group.

An interesting finding in our study was worsening in 'medication effects' in a higher percentage of Group 1 patients (31\%) as compared to Group 2 patients (14\%), even though the difference was not statistically significant. This worsening has not been reported by other authors. We postulate that all the patients in the preoperative period are too preoccupied with their seizures and its consequences on their emotional, social and overall functioning that they do not give importance to the adverse effects of medications. Once their seizures are controlled, some of them start worrying about the adverse effects of medications on their health.

\section{Conclusions}

Complete seizure-free state after surgery is associated with very significant improvement in HRQOL parameters. Several, but not all parameters of HRQOL as assessed by QOLIE-31, improve after surgery even in the poor seizure outcome group. The improvement in the domains of seizure worry, overall QOL, emotional wellbeing and social functioning is significantly more in those patients in whom complete seizure-free state is achieved.

\section{References}

1. Ryan R, Kempner K, E mlen AC. The stigma of epilepsy as a self concept. E pilepsia 1980;21:433-44.

2. Rausch $\mathrm{R}, \mathrm{Crandall} \mathrm{PH}$. Psychological status related to surgical control of temporal lobe seizures. E pilepsia 1982;23:191-202.

3. Calman KC. Quality of life in cancer patients: A hypothesis. J Med E thics 1984;10:124-7.

4. E ngel J J r. Outcome with respect to epileptic seizures. In: E ngel J J r, editor. Surgical treatment of epilepsies. Raven Press: N ew York; 1987.

5. Full QU OLIE-31 available from http://www.epilepsy.com/pdfs/qolie 31.pdf.

6. Cramer J A, Perrine K, Devinsky O, B ryant-Comstock L, Meador K, H ermann B. D evelopment and cross cultural translations of a 31 item quality of life in epilepsy inventory. E pilepsia 1998;39:81-8.

7. Golden $C J$, M oses J A, F ishburne F J, E ngum E, L ewis GP, Wisniewski $A M, \&$ al. Cross-validation of the $L$ uria-N ebraska N europsychological $B$ attery for the presence, lateralization and localization of brain damage. J Consult Clin Psychol 1981;49:491-507.

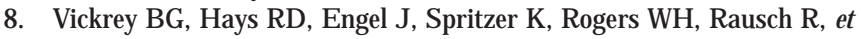
al. Outcome assessment for epilepsy surgery: The impact of measuring health-related quality of life. Ann N eurol 1995;37:158-66.

9. B irbeck GL, Sehyun K, H ays RD, Vickrey B G. Quality of life measures in epilepsy: How well can they detect change over time? Neurology 2000;54:1822-7.

10. Cramer J A, Arrigo C, H ammee GV, B romfield E B. Comparison between the QI L OE -31 and derived Q OL I E -10 in a clinical trial of levatiracetam. E pilepsy Res 2000;41:29-38.

11. Kim YH, Kim HI. Assessing quality of life for the measurement of outcome after epilepsy. surgery. Psychiatry Clin Neurosci 1995;49: 
S304-5.

12. Hermann BP, Wyler AR, Somes G. Preoperative psychological adjustments and surgical outcome are determinants of psychosocial status after anterior temporal lobectomy. J N eurol N eurosurg Psychiatry 1992;55:491-6.

13. Rose KJ , Derry PA, Wiebe S, MCL achian RS. Determinants of health related quality of life after temporal lobe epilepsy surgery. Qual L ife Res 1996;5:395-402.

14. Selai CE, E Istner K, Trimble MR. Quality of life pre and post epilepsy surgery. E pilepsy Res 2000;38:67-74.

15. Markand ON, Salanova V, Whelihan E, E msley CL. H ealth related quality of life outcome in medically refractory epilepsy treated with anterior temporal lobectomy. E pilepsia 2000;41:749-59.

16. K ellett MW, Smith DF, B aker GA, Chadwick DW. Quality of life after epilepsy surgery. J N eurol Neorosurg Psychiatry 1997;63:52-8.

17. Smith D, B aker GA, J acoby A, Chadwick DW. The contribution of the measurement of seizure severity to quality of life research. Qual L ife Res 1995;4:143-58.

18. Mihara $Y$, I noue $Y$, Watanabe $Y, M$ atsuda $K$, Tottori $T, H$ iyoshi $T$, $₫$ al. I mprovement in quality of life following resective surgery for temporal lobe epilepsy: Results of patients and family assessments. J pn J Psychiatry N eurol 1994:48:221-9.

19. Taylor DC, Falconer MA. Clinical, socio-economic and psychological changes after temporal lobectomy for epilepsy. B $\mathrm{r}$ J Psychiatry 1968;114:1247-61.

20. Seidman-Ripley J G, B ound VK, Andermann F, Olivier A, G loor P, F eindel WH . Psychosocial consequences of postoperative seizure relief. E pillepsia
1993;34:248-54.

21. B exendale SA, Thonpson PJ . "If I didn't have epilepsy...": Patient expectations of epilepsy surgery. J E pilepsy 1996;9:274-81.

22. Weiss AA. Criteria of prediction of successful rehabilitation after temporal lobectomy from pre-operative psychological investigation. I sr Ann Psychiatr Relat Discip 1965;3:65-72.

23. Vickrey BG, H ays RD, Graber J, Rausch R, Engel J, Brook RH. A health related quality of life instrument for patients evaluated for epilepsy surgery. Med Care 1992;30:299-319.

24. Vickrey B G, H ays RD, Rausch R, Suthering WW, Engel J E, B rok RH . Quality of life of epilepsy surgery patients as compared with outpatients with hypertension, diabetes, heart disease or depressive symptoms. E plilepsia 1994;35:597-607.

25. M rabet $H$, M rabet A, Zouari B, Ghachem R. H ealth-related quality of life of people with epilepsy compared with a general reference population: A Tunisian study. E pilepsia 2004;45:838-43.

26. B aker GA, J acoby A, Gorry J , D oughty J, E llina V; SI GN Group. Quality of life of people with epilepsy in I ran, the Gulf and N ear E ast. E pilepsia 2005;46:132-40.

27. Mikati MA, Comair YG, Rahi A. Normalization of quality of life three years after temporal lobectomy: A controlled study. E pilepsia 2006;47:928-33.

Accepted on 16-06-2007

Source of Support: Nil, Conflict of Interest: None declared. 